\title{
High-Frequency Electromagnetic Currents of Transition Region of an Impedance Circular Cylinder Illuminated by an Obliquely Incident Plane Wave
}

\author{
Non-member Kohei Hongo \\ (Faculty of Science, Toho University, hongo@is.sci.toho-u.ac.jp) \\ Non-member Hirokazu Kobayashi \\ (Fujitsu System Integration Laboratories, kobayasi@fsi.se.fujitsu.co.jp)
}

\begin{abstract}
A Fock-type representation of the surface fields can be obtained for the transition region of the circular cylinder with impedance boundary conditions when it is illuminated by an obliquely incident plane wave. First, the eigen function expansions for the surface fields are derived using a standard method. Then, the Watson transformation is applied to derive expressions which are uniform in the transition region. This current supplements the PO current approximation near the transition region. The correction to the PO currents is expected to improve upon the accuracy of the prediction of the scattered field. The validity of the Fock-type representation was verified numerically by comparing with the exact series solution.
\end{abstract}

\section{Keywords: Impedance Circular Cylinder, Obliquely Incident, Transition Current, Physical Optics}

\section{Introduction}

The physical optics (PO) approximation is known to be a useful approach for predicting a diffracted or radiated field, since it reduces the determination of the field to quadratures. Through the use of a radiation integral the PO also overcomes the drawback of the geometrical optics (GO) at caustics and shadow boundaries. However, the PO approximation does not always provide reliable solutions for the high-frequency fields, as the method still relies upon the GO approximation to determine the PO surface current. The surface currents obtained by the GO are in error near shadow boundaries on the surface of the scatterer. Within this so-called transition region, the $\mathrm{PO}$ current considerably deviates from the exact total current and this is clearly the main reason for errors in the $\mathrm{PO}$ predictions of the field scattered from smooth convex surfaces. Thus the accuracy of the computed scattered fields can be improved by accounting for the contribution to the scattered fields of the difference between the total and PO currents. The difference current, we call it transition current, can be derived from the exact solution to the canonical shape that conforms to the shape of the general scatterer in the vicinity of the shadow boundary.

Starting from a canonical problem of diffraction of a plane electromagnetic wave incident normally to the axis of an infinite circular cylinder with impedance boundary conditions, Osipov, et al, demonstrated that the transition current becomes particularly important for the accurate determination of the near fields, sidelobe fields, fields near null and the cross-polarized components of the scattered fields $[1,2]$.

The purpose of the present paper is to derive the transition current of an impedance circular cylinder illumi- nated by a plane wave incident obliquely to the cylinder axis. The analysis focuses on the development of a Fock-type asymptotic representation for electromagnetic field tangent components on the surface of the scatterer. This expression is very important to the high-frequency analysis since the use of the series expression is limited to some small values of $k a$, where $k a$ is the normalized radius of the cylinder. For a perfectly conducting cylinder surface, the role of transition current was analyzed by finding the far field contribution attributed to the $\mathrm{PO}$ and transition currents when a plane wave is incident normally to the cylinder, and the solution is generalized to that for arbitrary angles of incidence by a simple substitution method. For an impedance cylinder it is difficult to apply the substitution method to obtain the field for an arbitrary angle of incidence from the corresponding two dimensional solution. In contrast to the papers $[3,4]$ which treated the derivation of incremental length diffraction coefficients corresponding to an equivalent line source, we are concerned with the analysis of the real transition currents in a vicinity of the penumbra region on the surface of the scatterer. Once the transition currents, or the associated electromagnetic field tangent components, which are the same, are found, they can be directly inserted in the radiation integral to provide an improved solution to the problem of scattering from a smooth convex nonmetallic body at high-frequencies. The use of the real currents in the radiation integral allows one to obtain more accurate field representations, valid not only far from the cylinder but also at finite distance, including the surface of the cylinder. The validity of the Focktype representation of the surface fields is verified numerically by comparing with the series expression for various values of radius $k a$ and surface impedance. The 


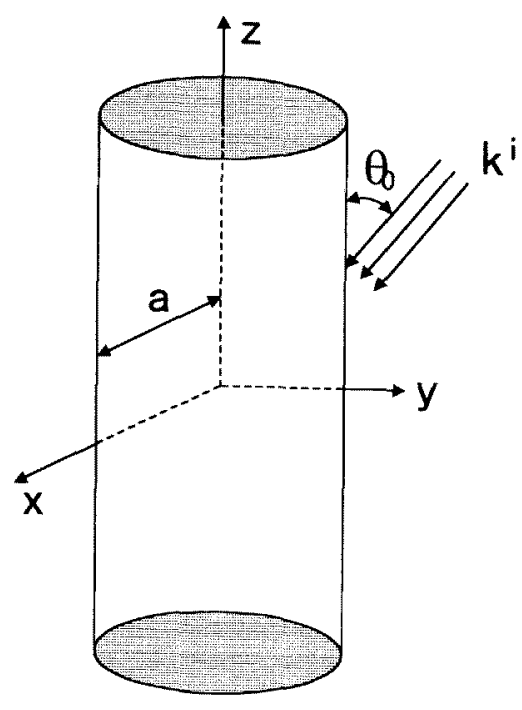

Fig. 1. Geometry of the problem

ıgreement is fairly good.

\section{Statement of the Problem}

The geometry of the problem and the associated cordinates $(\rho, \phi, z)$ are shown in Fig. 1, where the radius If the cylinder is $a$, angle of incidence is $\theta_{0}$, and wave rector $\mathbf{k}^{i}$ of an incident wave lies in the $x z$-plane. The ncident wave is given by

$$
\begin{aligned}
\mathbf{E}^{i}= & \left(A \mathbf{i}_{\theta}+B \mathbf{i}_{\phi}\right) \\
& \cdot \exp \left[j k\left(x \sin \theta_{0}+z \cos \theta_{0}\right)\right] \\
\mathbf{H}^{i}= & Y_{0}\left(-A \mathbf{i}_{\phi}+B \mathbf{i}_{\theta}\right) \\
& \cdot \exp \left[j k\left(x \sin \theta_{0}+z \cos \theta_{0}\right)\right], \\
\mathbf{k}^{i}= & \mathbf{i}_{x} \sin \theta_{0}+\mathbf{i}_{z} \cos \theta_{0} \ldots \ldots \ldots
\end{aligned}
$$

where

$$
\begin{aligned}
\mathbf{i}_{\theta} & =\cos \theta_{0} \mathbf{i}_{x}-\sin \theta_{0} \mathbf{i}_{z}, \\
\mathbf{i}_{\phi} & =\mathbf{i}_{y} \ldots \ldots \ldots \ldots \ldots
\end{aligned}
$$

ind $Y_{0}$ is an intrinsic admittance of free space. On the urface of the cylinder, the impedance boundary condiions

$$
\begin{aligned}
& E_{z}^{i}+E_{z}^{s}=\zeta\left[H_{\phi}^{i}+H_{\phi}^{s}\right], \\
& H_{z}^{i}+H_{z}^{s}=-\eta\left[E_{\phi}^{i}+E_{\phi}^{s}\right]
\end{aligned}
$$

aust be satisfied, where $\zeta$ and $\eta$ are surface impedance nd admittance of the scatterer, respectively, generlly $\zeta$ is not equal $1 / \eta$. Superscripts " $i$ " and " $s$ " resr to incident and scattered waves. We introduce the -components of magnetic and electric vector potenlals $A_{z}$ and $F_{z}$, respectively, which relate to the elecromagnetic field components by

$$
\begin{aligned}
\mathbf{E}= & -j \omega\left[A_{z} \mathbf{i}_{z}+\frac{1}{k^{2}} \frac{\partial}{\partial z} \nabla A_{z}\right] \\
& -\frac{1}{\epsilon} \nabla \times\left(\mathbf{i}_{z} F_{z}\right) \ldots \ldots \ldots
\end{aligned}
$$

$$
\begin{aligned}
\mathbf{H}= & -j \omega\left[F_{z} \mathbf{i}_{z}+\frac{1}{k^{2}} \frac{\partial}{\partial z} \nabla F_{z}\right] \\
& +\frac{1}{\mu} \nabla \times\left(\mathbf{i}_{z} A_{z}\right) \cdot \ldots \ldots
\end{aligned}
$$

The potential functions $A_{z}$ and $F_{z}$ corresponding to the incident and scattered fields can be expressed as follows:

$$
\begin{aligned}
A_{z}^{i}= & -\frac{j A}{\omega \sin \theta_{0}} \exp \left[j k z \cos \theta_{0}\right] \\
& \cdot \sum_{n=-\infty}^{\infty} j^{n} J_{n}\left(k \rho \sin \theta_{0}\right) \exp [-j n \phi] \cdots \cdots \\
F_{z}^{i}= & -\frac{j Y_{0} B}{\omega \sin \theta_{0}} \exp \left[j k z \cos \theta_{0}\right] \\
& \cdot \sum_{n=-\infty}^{\infty} j^{n} J_{n}\left(k \rho \sin \theta_{0}\right) \exp [-j n \phi] \cdots \cdots \\
A_{z}^{s}= & -\frac{j}{\omega \sin \theta_{0}} \exp \left[j k z \cos \theta_{0}\right] \\
& \cdot \sum_{n=-\infty}^{\infty} j^{n} a_{n} H_{n}^{(2)}\left(k \rho \sin \theta_{0}\right) \exp [-j n \phi] . \\
F_{z}^{s}= & -\frac{j Y_{0}}{\omega \sin \theta_{0}} \exp \left[j k z \cos \theta_{0}\right] \\
& \cdot \sum_{n=-\infty}^{\infty} j^{n} b_{n} H_{n}^{(2)}\left(k \rho \sin \theta_{0}\right) \exp [-j n \phi] \quad
\end{aligned}
$$

where formula of the wave transformation

$$
\exp (j k x)=\sum_{n=-\infty}^{\infty} j^{n} J_{n}(k \rho) \exp [-j n \phi]
$$

is used. In the above equation, $J_{n}(x)$ and $H_{n}^{(2)}(x)$ are the Bessel and the second kind of Hankel function of order $n$ and argument $x$, respectively. Symbols $a_{n}$ and $b_{n}$ stand for the expansion coefficients which are to be determined so that the total fields satisfy the required boundary conditions. The tangent magnetic components on the surface are given by

$$
\begin{aligned}
H_{z}^{t}= & Y_{0} A \frac{2 \zeta_{0} \eta_{0}}{\pi \Lambda} \cos \theta_{0} \exp \left[j k z \cos \theta_{0}\right] \\
& \cdot \sum_{n=-\infty}^{\infty} j^{n} \frac{n}{k a} \frac{H_{n}^{(2)}(\Lambda)}{\Delta(n)} \exp [-j n \phi] \\
& -Y_{0} B \frac{2 \eta_{0}}{\pi \Lambda} \sin \theta_{0} \exp \left[j k z \cos \theta_{0}\right] \\
& \cdot \sum_{n=-\infty}^{\infty} j^{n} \frac{j \zeta_{0} H_{n}^{(2) \prime}(\Lambda)+\sin \theta_{0} H_{n}^{(2)}(\Lambda)}{\Delta(n)} \\
& \cdot \exp [-j n \phi] \ldots \ldots \ldots \ldots \ldots \ldots
\end{aligned}
$$

$$
\begin{aligned}
H_{\phi}^{t}= & -Y_{0} A \frac{2}{\pi \Lambda} \sin \theta_{0} \exp \left[j k z \cos \theta_{0}\right] \\
& \cdot \sum_{n=-\infty}^{\infty} j^{n} \frac{j \eta_{0} H_{n}^{(2)}(\Lambda)+\sin \theta_{0} H_{n}^{(2)}(\Lambda)}{\Delta(n)} \\
& \cdot \exp [-j n \phi]
\end{aligned}
$$




$$
\begin{aligned}
& -Y_{0} B \frac{2 \eta_{0}}{\pi \Lambda} \cos \theta_{0} \exp \left[j k z \cos \theta_{0}\right] \\
& \cdot \sum_{n=-\infty}^{\infty} j^{n} \frac{n}{k a} \frac{H_{n}^{(2)}(\Lambda)}{\Delta(n)} \exp [-j n \phi] \cdots \\
\Delta(n)= & {\left[j \zeta_{0} H_{n}^{(2) \prime}(\Lambda)+\sin \theta_{0} H_{n}^{(2)}(\Lambda)\right] } \\
& \cdot\left[j \eta_{0} H_{n}^{(2) \prime}(\Lambda)+\sin \theta_{0} H_{n}^{(2)}(\Lambda)\right] \\
& +\zeta_{0} \eta_{0} \frac{\cos ^{2} \theta_{0}}{\sin ^{2} \theta_{0}}\left[\frac{n}{k a} H_{n}^{(2)}(\Lambda)\right]^{2} \ldots \ldots
\end{aligned}
$$

where $\Lambda=k a \sin \theta_{0}$. The derivation of these equations is shown in Appendix A. Since $E_{z}^{t}$ and $E_{\phi}^{t}$ are related to $H_{\phi}^{t}$ and $H_{x}^{t}$ by Eq. (4) on the surface, these components can be omitted.

For the special case of a perfect conductor, these expressions are simplified when the limitting process $\zeta_{0} \rightarrow 0, \eta_{0} \rightarrow \infty$, and $\eta_{0} \zeta_{0} \rightarrow 1$ are applied. The result is

$$
\begin{aligned}
H_{z}^{t}= & \frac{j 2 Y_{0} B}{\pi k a} \exp \left[j k z \cos \theta_{0}\right] \\
& \cdot \sum_{n=-\infty}^{\infty} j^{n} \frac{\exp [-j n \phi]}{H_{n}^{(2)}\left(k a \sin \theta_{0}\right)} \cdots \\
H_{\phi}^{t}= & \frac{2 Y_{0} A}{\pi k a \sin \theta_{0}} \exp \left[j k z \cos \theta_{0}\right] \\
& \cdot \sum_{n=-\infty}^{\infty} j^{n} \frac{\exp [-j n \phi]}{H_{n}^{(2)}\left(k a \sin \theta_{0}\right)} \\
& +\frac{j 2 Y_{0} B}{\pi(k a)^{2}} \frac{\cos \theta_{0}}{\sin ^{2} \theta_{0}} \exp \left[j k z \cos \theta_{0}\right] \\
& \cdot \sum_{n=-\infty}^{\infty} j^{n} \frac{n \exp [-j n \phi]}{H_{n}^{(2)}\left(k a \sin \theta_{0}\right)} \ldots
\end{aligned}
$$

It is noted that the expression $H_{z}^{t}$ and the first term of $H_{\phi}^{t}$ are same forms of normal incidence except that the argument $k a$ of the Hankel function has been replaced by $k a \sin \theta_{0}$, and the second term of $H_{\phi}^{t}$ was obtained from $H_{z}^{t}$ by performing differentiation, with respect to $\phi$. This is the reason that these expressions can be derived from the corresponding normal incident solution by the substitution method.

In contrast to a perfectly conducting cylinder, it is difficult to construct the solution with this technique. It is readily seen that the tangential components $H_{z}^{t}$ and $H_{\phi}^{t}$ consist of two kinds of series given by

$$
\begin{aligned}
U(\phi) & =\sum_{n=-\infty}^{\infty} j^{n} \frac{H_{n}^{(2)}(\Lambda)}{\Delta(n)} \exp [-j n \phi] \\
& =\sum_{n=-\infty}^{\infty} j^{n} \frac{n}{k a \sin \theta_{0}} \frac{H_{n}^{(2)}(\Lambda)}{\Delta(n)} \exp [-j n \phi] \\
V(\phi) & =\sum_{n=-\infty}^{\infty} j^{n} \frac{H_{n}^{(2) \prime}(\Lambda)}{\Delta(n)} \exp [-j n \phi] \ldots \ldots
\end{aligned}
$$

\section{High-Frequency Representations of the Surface Fields}

When we consider the diffraction of the electromagnetic wave by a smooth obstacle, the equivalent radius of curvature varies over a wide range. Hence the approximate solution is essential to this kind of problem. In case the radius of the circular cylinder becomes very large compared with the wavelength, the series representations are inadequate and these are transformed into more useful expressions using the Watson transformation. The detail of the derivation is given in Appendix $\mathrm{B}$.

(a) Perfectly Conducting Circular Cylinder

$$
\begin{aligned}
H_{z}^{t}= & -Y_{0} B \sin \theta_{0} \\
& \cdot \exp \left[j k z \cos \theta_{0}-j k a \sin \theta_{0}\left(\phi-\frac{\pi}{2}\right)\right] \\
& \cdot g\left[m\left(\phi-\frac{\pi}{2}\right)\right] \ldots \ldots \ldots \ldots \ldots \ldots(19) \\
H_{\phi}^{t}= & -Y_{0} B \cos \theta_{0} \\
& \cdot \exp \left[j k z \cos \theta_{0}-j k a \sin \theta_{0}\left(\phi-\frac{\pi}{2}\right)\right] \\
& \cdot g\left[m\left(\phi-\frac{\pi}{2}\right)\right] \\
& -\frac{Y_{0} B}{m} \exp \left[j k z \cos \theta_{0}-j k a \sin \theta_{0}\left(\phi-\frac{\pi}{2}\right)\right] \\
& \cdot f\left[m\left(\phi-\frac{\pi}{2}\right)\right] \ldots \ldots \ldots \ldots \ldots(20)
\end{aligned}
$$

(b) Impedance Circular Cylinder

$$
\begin{aligned}
H_{z}^{t}= & Y_{0} A \frac{2 \zeta_{0} \eta_{0}}{\pi k a} \cos \theta_{0} \exp \left[j k z \cos \theta_{0}\right] U(\phi) \\
& -Y_{0} B \frac{2 \eta_{0}}{\pi k a} \exp \left[j k z \cos \theta_{0}\right] \\
& \cdot\left[j \zeta_{0} V(\phi)+\sin \theta_{0} U(\phi)\right] \\
H_{\phi}^{t}= & -Y_{0} A \frac{2}{\pi k a} \exp \left[j k z \cos \theta_{0}\right] \\
& \cdot\left[j \eta_{0} V(\phi)+\sin \theta_{0} U(\phi)\right] \\
& -Y_{0} B \frac{2 \eta_{0}}{\pi k a} \cos \theta_{0} \exp \left[j k z \cos \theta_{0}\right] U(\phi)
\end{aligned}
$$

In the above equations, the functions $f(\xi), g(\xi), U(\phi)$, and $V(\phi)$ are defined in Appendix B.

The Fock-type expressions for the function $U(\phi)$ and $V(\phi)$ defined by $\mathrm{Eq} \cdot(17),(18)$ and (A27)-(A30) are programmed and compared to various values of $k a$ and surface impedance. Tab.1 shows some illustrating results for parameters $k a=50, \theta_{0}=60^{\circ}, \zeta_{0}=0.5-j 0.5$, $\eta_{0}=\frac{1}{\zeta_{0}}$, and $A=1, B=0$. It can be seen that the agreement is fairly good.

\section{Conclusion}

A Fock-type representation of the surface fields was obtained for the transition region of the circular cylinder with impedance boundary conditions when it is illuminated by an obliquely incident plane wave. The 
Table 1. Comparison of the surface fields computed from series (exact) and asymptotic (Fock-type) expressions. The parameters are: $k a=50, \theta_{0}=60^{\circ}, \zeta_{0}=0.5-j 0.5, \eta_{0}=1 / \zeta_{0}, A=1, B=0$.

\begin{tabular}{|c|c|c|c|c|c|c|c|c|c|}
\hline$\phi$ & $\mathrm{H}_{2}$ (Exact) & $\mathrm{H}_{2}$ (Approx) & $H_{\phi}$ (Exact) & $H_{\phi}$ (Approx) & $\phi$ & $\mathrm{H}_{\mathrm{z}}$ (Exact) & $\mathrm{H}_{\mathrm{z}}$ (Approx) & $H_{\phi}$ (Exact) & $H_{\phi}$ (Approx) \\
\hline 70 & 0.19196 & 0.20139 & 0.53944 & 0.53680 & 96 & 0.08753 & 0.08420 & 0.15501 & 0.15734 \\
\hline 71 & 0.18952 & 0.19760 & 0.52211 & 0.51945 & 97 & 0.08325 & 0.08001 & 0.14516 & 0.14761 \\
\hline 72 & 0.18688 & 0.19370 & 0.50480 & 0.50216 & 98 & 0.07906 & 0.07594 & 0.13577 & 0.13832 \\
\hline 73 & 0.18404 & 0.18968 & 0.48752 & 0.48495 & 99 & 0.07497 & 0.07197 & 0.12682 & 0.12946 \\
\hline 74 & 0.18101 & 0.18555 & 0.47030 & 0.46783 & 100 & 0.07100 & 0.06813 & 0.11832 & 0.12102 \\
\hline 75 & 0.17779 & 0.18131 & 0.45317 & 0.45084 & 101 & 0.06714 & 0.06441 & 0.11025 & 1300 \\
\hline 76 & 0.17440 & 0.17699 & 0.43616 & 0.43398 & 102 & 0.06340 & 0.06081 & 0.10261 & 539 \\
\hline 77 & 0.17084 & 0.17257 & 0.41928 & 0.41729 & 103 & 0.05979 & 0.05735 & 539 & 819 \\
\hline 78 & 0.16713 & 0.16807 & 0.40257 & 0.40079 & 104 & 0.05630 & 5401 & 857 & 09138 \\
\hline 79 & 0.16326 & 0.16349 & 0.38605 & 0.38450 & 105 & 0.05296 & 0.05081 & 0.08215 & 0.08495 \\
\hline 80 & 0.15926 & 0.15886 & 0.36974 & 0.36843 & 106 & 0.04974 & 0.04774 & 0.07612 & 0.07889 \\
\hline 81 & 0.15514 & 0.15416 & 0.35368 & 0.35262 & 107 & 0.04667 & 0.04480 & 0.07045 & 0.07319 \\
\hline 82 & 0.15090 & 0.14943 & 0.33788 & 0.33708 & 108 & 0.04373 & 0.04200 & 0.06514 & 0.06784 \\
\hline 83 & 0.14657 & 0.14465 & 0.32236 & 0.32184 & 109 & 0.04092 & 0.03933 & 017 & .06282 \\
\hline 84 & 0.14215 & 0.13986 & 0.30716 & 0.30690 & 110 & 0.03826 & 3679 & 553 & 5811 \\
\hline 85 & 0.13765 & 0.13504 & 0.29229 & 0.29230 & 111 & 0.03572 & 0.03438 & 5120 & 5372 \\
\hline 86 & 0.13311 & 0.13023 & 0.27777 & 0.27805 & 112 & 0.03332 & 0.03209 & 7717 & 4961 \\
\hline 87 & 0.12851 & 0.12542 & 0.26361 & 0.26415 & 113 & 0.03105 & 0.02993 & 342 & 0.04578 \\
\hline 88 & 0.12389 & 0.12063 & 0.24985 & 0.25064 & 114 & 0.02890 & 788 & 0.03994 & 0.04222 \\
\hline 89 & 0.11926 & 0.11587 & 0.23648 & 0.23752 & 115 & 0.02688 & 0.02595 & 0.03671 & 0.03891 \\
\hline 90 & 0.11462 & 0.11115 & 0.22353 & 0.22479 & 116 & 0.02497 & 0.02413 & 0.03372 & 0.03583 \\
\hline 91 & 0.11000 & 0.10648 & 0.21100 & 0.21248 & 117 & 0.02318 & 0.02242 & 0.03095 & 0.03297 \\
\hline 92 & 0.10540 & 0.10187 & 0.19891 & 0.20059 & 118 & 0.02150 & 0.02082 & 0.02840 & 0.03032 \\
\hline 93 & 0.10085 & 0.09733 & 0.18726 & 0.18913 & 119 & 0.01992 & 0.01931 & 0.02604 & 0.02788 \\
\hline 94 & 0.09634 & 0.09286 & 0.17605 & 0.17810 & 120 & 0.01845 & 0.01790 & 0.02386 & 0.02561 \\
\hline 95 & 0.09190 & 0.08848 & 0.16530 & 0.16750 & & & & & \\
\hline
\end{tabular}

validity of the Fock-type representation was verified numerically by comparing it with the exact series solution. The application of this expression to predict the fields diffracted by various kinds of obstacles is under study. 99)

(Manuscript received October 2, 98, revised April 9,

\section{References}

(1) V. A. Fock, Electromagnetic Diffraction and Propagation Problems, Pergamon, New York, 1965.

(2) A. V. Osipov, H. Kobayashi and K. Hongo, Shadow Boundary Currents in the Problem of High-Frequency Electromagnetic Diffraction by a Circular Impedance Cylinder, IEICE Trans. Electronics, VOL.E81-C, NO.10, pp1655-1666, Oct., 1998.

(3) A. D. Yaghjian, R. A. Shore and M. B. Woodworth, Shadow Boundary Incremental Length Diffraction Coefficients for Perfectly Conducting Smooth, Convex Surface, Radio Science vol. 31 , pp.1681-1695, 1996.

(4) T. B. Hansen and R. A. Shore, Incremental Length Diffraction Coefficients for the Shadow Boundary of Convex Cylinder, to be appeared in IEEE Trans. on Antennas and Propagation.

\section{Appendix}

\section{A-1. The Surface Fields on the Impedance Cylinder}

In this appendix, the detailed derivation of the surface fields on the impedance cylinder is shown. The explicit expressions for incident and scattered fields are given as follows:

$$
\begin{aligned}
& E_{z}^{i}=-A \sin \theta_{0} \exp \left[j k z \cos \theta_{0}\right] \\
& \sum_{n=-\infty}^{\infty} j^{n} J_{n}\left(k \rho \sin \theta_{0}\right) \exp [-j n \phi] \ldots \\
& H_{z}^{i}=-Y_{0} B \sin \theta_{0} \exp \left[j k z \cos \theta_{0}\right] \\
& \text {. } \sum_{n=-\infty}^{\infty} j^{n} J_{n}\left(k \rho \sin \theta_{0}\right) \exp [-j n \phi] \ldots \\
& E_{\phi}^{i}=-\frac{A \cos \theta_{0}}{\sin \theta_{0}} \cdot \exp \left[j k z \cos \theta_{0}\right] \\
& \text {. } \sum_{n=-\infty}^{\infty} j^{n} \frac{n}{k \rho} J_{n}\left(k \rho \sin \theta_{0}\right) \exp [-j n \phi] \\
& -j B \exp \left[j k z \cos \theta_{0}\right] \\
& \text {. } \sum_{n=-\infty}^{\infty} j^{n} J_{n}^{\prime}\left(k \rho \sin \theta_{0}\right) \exp [-j n \phi] \cdots \\
& H_{\phi}^{i}=-\frac{Y_{0} B \cos \theta_{0}}{\sin \theta_{0}} \exp \left[j k z \cos \theta_{0}\right] \\
& \text {. } \sum_{n=-\infty}^{\infty} j^{n} \frac{n}{k \rho} J_{n}\left(k \rho \sin \theta_{0}\right) \exp [-j n \phi] \\
& +j Y_{0} A \exp \left[j k z \cos \theta_{0}\right] \\
& \text {. } \sum_{n=-\infty}^{\infty} j^{n} J_{n}^{\prime}\left(k \rho \sin \theta_{0}\right) \exp [-j n \phi] \ldots \\
& E_{z}^{s}=-\sin \theta_{0} \exp \left[j k z \cos \theta_{0}\right] \\
& \text {. } \sum_{n=-\infty}^{\infty} j^{n} a_{n} H_{n}^{(2)}\left(k \rho \sin \theta_{0}\right) \exp [-j n \phi] \\
& H_{z}^{s}=-Y_{0} \sin \theta_{0} \exp \left[j k z \cos \theta_{0}\right]
\end{aligned}
$$




$$
\begin{aligned}
& \cdot \sum_{n=-\infty}^{\infty} j^{n} b_{n} H_{n}^{(2)}\left(k \rho \sin \theta_{0}\right) \exp [-j n \phi] \quad \text { (A6) } \\
E_{\phi}^{s}= & -\frac{\cos \theta_{0}}{\sin \theta_{0}} \cdot \exp \left[j k z \cos \theta_{0}\right] \\
& \cdot \sum_{n=-\infty}^{\infty} j^{n} a_{n} \frac{n}{k \rho} H_{n}^{(2)}\left(k \rho \sin \theta_{0}\right) \exp [-j n \phi] \\
& -j \exp \left[j k z \cos \theta_{0}\right] \sum_{n=-\infty}^{\infty} j^{n} b_{n} H_{n}^{(2) \prime}\left(k \rho \sin \theta_{0}\right) \\
& \cdot \exp [-j n \phi] \ldots \ldots \ldots \ldots \text { (A } 7) \\
H_{\phi}^{s}= & -Y_{0} \frac{\cos \theta_{0}}{\sin \theta_{0}} \cdot \exp \left[j k z \cos \theta_{0}\right] \\
& \cdot \sum_{n=-\infty}^{\infty} j^{n} b_{n} \frac{n}{k \rho} H_{n}^{(2)}\left(k \rho \sin \theta_{0}\right) \exp [-j n \phi] \\
+ & j Y_{0} \exp \left[j k z \cos \theta_{0}\right] \sum_{n=-\infty}^{\infty} j^{n} a_{n} H_{n}^{(2) \prime}\left(k \rho \sin \theta_{0}\right) \\
& \cdot \exp [-j n \phi] \ldots \ldots \ldots \ldots \text { (A } 8)
\end{aligned}
$$

where superscripts " $i$ " refers to the incident wave and " $s$ " refers to the scattered field, respectively. Enforcement of the boundary condition yields the equation for the unknown expansion coefficients $a_{n}$ and $b_{n}$.

$$
\begin{aligned}
& {\left[j \zeta_{0} H_{n}^{(2) \prime}(\Lambda)+\sin \theta_{0} H_{n}^{(2)}(\Lambda)\right] a_{n}} \\
& -\zeta_{0} \frac{\cos \theta_{0}}{\sin \theta_{0}} \frac{n}{k a} H_{n}^{(2)}(\Lambda) b_{n} \\
& =-A\left[\sin \theta_{0} J_{n}(\Lambda)+j \zeta_{0} J_{n}{ }^{\prime}(\Lambda)\right] \\
& \quad+B \zeta_{0} \frac{\cos \theta_{0}}{\sin \theta_{0}} \frac{n}{k a} J_{n}(\Lambda) \ldots \ldots \ldots \\
& \eta_{0} \frac{\cos \theta_{0}}{\sin \theta_{0}} \frac{n}{k a} H_{n}^{(2)}(\Lambda) a_{n} \\
& +\left[j \eta_{0} H_{n}^{(2) \prime}(\Lambda)+\sin \theta_{0} H_{n}^{(2)}(\Lambda)\right] b_{n} \\
& \quad=-A \eta_{0} \frac{\cos \theta_{0}}{\sin \theta_{0}} \frac{n}{k a} J_{n}(\Lambda) \\
& \quad-B\left[\sin \theta_{0} J_{n}(\Lambda)+j \eta_{0} J_{n}{ }^{\prime}(\Lambda)\right]
\end{aligned}
$$

where $\Lambda=k a \sin \theta_{0}, \zeta_{0}=Y_{0} \zeta$ and $\eta_{0}=Z_{0} \eta$. The solution of these equations is given by

$$
\begin{aligned}
a_{n}= & \frac{1}{\Delta(n)}\left\{-\left[A\left[\sin \theta_{0} J_{n}(\Lambda)+j \zeta_{0} J_{n}{ }^{\prime}(\Lambda)\right]\right.\right. \\
& \left.-B \zeta_{0} \frac{\cos \theta_{0}}{\sin \theta_{0}} \frac{n}{k a} J_{n}(\Lambda)\right] \\
& \cdot\left[j \eta_{0} H_{n}^{(2) \prime}(\Lambda)+\sin \theta_{0} H_{n}^{(2)}(\Lambda)\right] \\
& -\left[A \eta_{0} \frac{\cos \theta_{0}}{\sin \theta_{0}} \frac{n}{k a} J_{n}(\Lambda)\right. \\
& \left.+B\left[\sin \theta_{0} J_{n}(\Lambda)+j \eta_{0} J_{n}{ }^{\prime}(\Lambda)\right]\right] \\
& \left.\cdot \zeta_{0} \frac{\cos \theta_{0}}{\sin \theta_{0}} \frac{n}{k a} H_{n}^{(2)}(\Lambda)\right\} \ldots \ldots(\mathrm{A} \\
b_{n}= & \frac{1}{\Delta(n)}\left\{\left[A\left[\sin \theta_{0} J_{n}(\Lambda)+j \zeta_{0} J_{n}{ }^{\prime}(\Lambda)\right]\right.\right.
\end{aligned}
$$

$$
\begin{aligned}
& \left.-B \zeta_{0} \frac{\cos \theta_{0}}{\sin \theta_{0}} \frac{n}{k a} J_{n}(\Lambda)\right] \\
& \cdot \eta_{0} \frac{\cos \theta_{0}}{\sin \theta_{0}} \frac{n}{k a} H_{n}^{(2)}(\Lambda) \\
& -\left[A \eta_{0} \frac{\cos \theta_{0}}{\sin \theta_{0}} \frac{n}{k a} J_{n}(\Lambda)\right. \\
& \left.+B\left[\sin \theta_{0} J_{n}(\Lambda)+j \eta_{0} J_{n}^{\prime}(\Lambda)\right]\right] \\
& \left.\cdot\left[j \zeta_{0} H_{n}^{(2) \prime}(\Lambda)+\sin \theta_{0} H_{n}^{(2)}(\Lambda)\right]\right\} \\
\Delta(n)= & {\left[j \zeta_{0} H_{n}^{(2) \prime}(\Lambda)+\sin \theta_{0} H_{n}^{(2)}(\Lambda)\right] } \\
& \cdot\left[j \eta_{0} H_{n}^{(2) \prime}(\Lambda)+\sin \theta_{0} H_{n}^{(2)}(\Lambda)\right] \\
& +\zeta_{0} \eta_{0} \frac{\cos ^{2} \theta_{0}}{\sin ^{2} \theta_{0}}\left[\frac{n}{k a} H_{n}^{(2)}(\Lambda)\right]^{2} . \cdots
\end{aligned}
$$

Using these results we can obtain the expressions for the surface fields

$$
\begin{aligned}
E_{z}^{t}= & -A \frac{2 \zeta_{0}}{\pi \Lambda} \sin \theta_{0} \exp \left[j k z \cos \theta_{0}\right] \\
& \cdot \sum_{n=-\infty}^{\infty} j^{n} \frac{j \eta_{0} H_{n}^{(2) \prime}(\Lambda)+\sin \theta_{0} H_{n}^{(2)}(\Lambda)}{\Delta(n)} \\
& \cdot \exp [-j n \phi] \\
& -B \frac{2 \zeta_{0} \eta_{0}}{\pi \Lambda} \cos \theta_{0} \exp \left[j k z \cos \theta_{0}\right] \\
& \cdot \sum_{n=-\infty}^{\infty} j^{n} \frac{n}{k a} \frac{H_{n}^{(2)}(\Lambda)}{\Delta(n)} \exp [-j n \phi] \cdots \\
H_{z}^{t}= & Y_{0} A \frac{2 \zeta_{0} \eta_{0}}{\pi \Lambda} \cos \theta_{0} \exp \left[j k z \cos \theta_{0}\right] \\
& \cdot \sum_{n=-\infty}^{\infty} j^{n} \frac{n}{k a} \frac{H_{n}^{(2)}(\Lambda)}{\Delta(n)} \exp [-j n \phi] \\
& -Y_{0} B \frac{2 \eta_{0}}{\pi \Lambda} \sin \theta_{0} \exp \left[j k z \cos \theta_{0}\right] \\
& \cdot \sum_{n=-\infty}^{\infty} j^{n} \frac{j \zeta_{0} H_{n}^{(2) \prime}(\Lambda)+\sin \theta_{0} H_{n}^{(2)}(\Lambda)}{\Delta(n)} \\
& \cdot \exp [-j n \phi] \cdots \cdots
\end{aligned}
$$

where Wronski's relation

$$
J_{n}(x) H_{n}^{(2) \prime}(x)-J_{n}^{\prime}(x) H_{n}^{(2)}(x)=-j \frac{2}{\pi x} \cdots
$$

is used to derive the above equation. Other tangential components $E_{\phi}^{t}$ and $H_{\phi}^{t}$ can be directly obtained from Eq.(A14),(A15) by using relation Eq.(4).

\section{A-2. Fock-type Representation of the Sur- face Fields}

From the discussion of Sec.2, the surface fields are obtained from four kinds of functions, $F(\phi), G(\phi), U(\phi)$, and $V(\phi)$ which are given by an infinite series. In this appendix, Fock-type representations of these functions are determined using Watson transformation.

(a) Functions $F(\phi)$ and $G(\phi)$ : 
$F(\phi)$ and $G(\phi)$ are defined as infinite series parts of Eq.(15),(16) and represented by Fock-type functions $f(\xi), g(\xi)$ as follows:

$$
\begin{aligned}
F(\phi)= & \sum_{n=-\infty}^{\infty} j^{n} \frac{\exp [-j n \phi]}{H_{n}^{(2)}\left(k a \sin \theta_{0}\right)} \\
= & \frac{1}{2 j} \int_{C} \frac{\exp [-j \nu(\phi-\pi)]}{\sin \nu \pi} \\
& \frac{\exp \left[j \frac{\nu \pi}{2}\right]}{H_{\nu}^{(2)}\left(k a \sin \theta_{0}\right)} d \nu \ldots .
\end{aligned}
$$

where $C$ is the contour which encircle the real axis counterclockwise. This can be transformed into

$$
\begin{aligned}
F(\phi)= & \frac{1}{2 j} \int_{-\infty-j \epsilon}^{\infty-j \epsilon} \frac{\exp [-j \nu(\phi-\pi)]}{\sin \nu \pi} \\
& \frac{\exp \left[j \frac{\nu \pi}{2}\right]}{H_{\nu}^{(2)}\left(k a \sin \theta_{0}\right)} d \nu \\
& +\frac{1}{2 j} \int_{-\infty-j \epsilon}^{\infty-j \epsilon} \frac{\exp [j \nu(\phi-\pi)]}{\sin \nu \pi} \\
& \cdot \frac{\exp \left[j \frac{\nu \pi}{2}\right]}{H_{\nu}^{(2)}\left(k a \sin \theta_{0}\right)} d \nu \\
= & -j \int_{-\infty-j \epsilon}^{\infty-j \epsilon} \frac{\cos [\nu(\phi-\pi)]}{\sin \nu \pi} \\
& \cdot \frac{\exp \left[j \frac{\nu \pi}{2}\right]}{H_{\nu}^{(2)}\left(k a \sin \theta_{0}\right)} d \nu \ldots \ldots \ldots
\end{aligned}
$$

when $k a \sin \theta_{0} \gg 1$, the main contribution comes from

$$
\frac{\cos \nu(\phi-\pi)}{\sin \nu \pi} \simeq j \exp (-j \nu \phi) \text {. }
$$

In a transition region, since the values near $k a$ contribute most significantly to the above integrals when $k a$ is very large, we can write

$$
\nu=k a \sin \theta_{0}+m \tau, \quad m=\left(\frac{k a \sin \theta_{0}}{2}\right)^{\frac{1}{3}} \text {. }
$$

In this region, the Hankel functions may be approximated by

$$
\begin{aligned}
& H_{\nu}^{(2)}\left(k a \sin \theta_{0}\right) \simeq \frac{j}{\sqrt{\pi}} \frac{1}{m} w_{2}(\tau), \\
& H_{\nu}^{(2) \prime}\left(k a \sin \theta_{0}\right) \simeq-\frac{j}{\sqrt{\pi}} \frac{1}{m^{2}} w_{2}^{\prime}(\tau) .
\end{aligned}
$$

Then, $F(\phi)$ and $G(\phi)$ are simplified as follows.

$$
\begin{aligned}
F(\phi)= & -j \pi m^{2} \exp \left[-j k a \sin \theta_{0}\left(\phi-\frac{\pi}{2}\right)\right] \\
& \cdot f\left[m\left(\phi-\frac{\pi}{2}\right)\right] \ldots \ldots \ldots \ldots \ldots
\end{aligned}
$$

where

$$
f(\xi)=\frac{1}{\sqrt{\pi}} \int_{-\infty}^{\infty} \frac{\exp [-j \xi t]}{w_{2}(t)} d t
$$

$$
\begin{aligned}
G(\phi)= & \sum_{n=-\infty}^{\infty} j^{n} \frac{\exp [-j n \phi]}{H_{n}^{(2) \prime}\left(k a \sin \theta_{0}\right)} \\
= & \sum_{n=-\infty}^{\infty} j^{n} \frac{n}{k a \sin \theta_{0}} \frac{\exp [-j n \phi]}{H_{n}^{(2) \prime}\left(k a \sin \theta_{0}\right)} \\
= & j \pi m^{3} \exp \left[-j k a \sin \theta_{0}\left(\phi-\frac{\pi}{2}\right)\right] \\
& \cdot g\left[m\left(\phi-\frac{\pi}{2}\right)\right] \ldots \ldots \ldots \ldots \ldots
\end{aligned}
$$

where

$$
g(\xi)=\frac{1}{\sqrt{\pi}} \int_{-\infty}^{\infty} \frac{\exp [-j \xi t]}{w_{2}^{\prime}(t)} d t
$$

The function $w_{2}(t)$ and $w_{2}^{\prime}(t)$ are Fock-type Airy function defined below and its first derivative, respectively.

$$
w_{1,2}(t)=\frac{1}{\pi} \int_{\Gamma_{1}, \Gamma_{2}} \exp \left[t z-\frac{1}{3} z^{3}\right] d z
$$

(b) Functions $U(\phi)$ and $V(\phi)$ :

$$
\begin{aligned}
U(\phi)= & \sum_{n=-\infty}^{\infty} j^{n} \frac{H_{n}^{(2)}\left(k a \sin \theta_{0}\right)}{\Delta(n)} \exp [-j n \phi] \\
= & \sum_{n=-\infty}^{\infty} j^{n} \frac{n}{k a \sin \theta} \frac{H_{n}^{(2)}\left(k a \sin \theta_{0}\right)}{\Delta(n)} \\
& \cdot \exp [-j n \phi] \\
= & -j \sqrt{\pi} m^{3} \exp \left[-j k a \sin \theta_{0}\left(\phi-\frac{\pi}{2}\right)\right] \\
& \cdot \int_{-\infty}^{\infty} \frac{w_{2}(t)}{D_{2}(t)} \exp \left[-j m t\left(\phi-\frac{\pi}{2}\right)\right] d t \\
= & -j \sqrt{\pi} m^{3} \exp \left[-j k a \sin \theta_{0}\left(\phi-\frac{\pi}{2}\right)\right] \\
& \cdot \int_{0}^{\infty} \frac{w_{2}(t)}{D_{2}(t)} \exp \left[-j m t\left(\phi-\frac{\pi}{2}\right)\right] d t \\
& +\exp \left(j \frac{\pi}{6}\right) \sqrt{\pi} m^{3} \\
& \cdot \exp \left[-j k a \sin \theta_{0}\left(\phi-\frac{\pi}{2}\right)\right] \int_{0}^{\infty} \frac{w_{1}(t)}{D_{1}(t)} \\
& \left.\cdot \exp \left[j m t\left(\phi-\frac{\pi}{2}\right)\left(\frac{1}{2}+j \frac{\sqrt{3}}{2}\right)\right] d t \cdot\left(A^{2}\right)\right]
\end{aligned}
$$

$$
\begin{aligned}
V(\phi)= & \sum_{n=-\infty}^{\infty} j^{n} \frac{H_{n}^{(2) \prime}\left(k a \sin \theta_{0}\right)}{\Delta(n)} \exp [-j n \phi] \\
= & j \sqrt{\pi} m^{2} \exp \left[-j k a \sin \theta_{0}\left(\phi-\frac{\pi}{2}\right)\right] \\
& \cdot \int_{-\infty}^{\infty} \frac{w_{2}^{\prime}(t)}{D_{2}(t)} \exp \left[-j m t\left(\phi-\frac{\pi}{2}\right)\right] d t \\
= & j \sqrt{\pi} m^{2} \exp \left[-j k a \sin \theta_{0}\left(\phi-\frac{\pi}{2}\right)\right] \\
& \cdot \int_{0}^{\infty} \frac{w_{2}^{\prime}(t)}{D_{2}(t)} \exp \left[-j m t\left(\phi-\frac{\pi}{2}\right)\right] d t \\
& +\exp \left(-j \frac{\pi}{6}\right) \sqrt{\pi} m^{2} \\
& \cdot \exp \left[-j k a \sin \theta_{0}\left(\phi-\frac{\pi}{2}\right)\right] \int_{0}^{\infty} \frac{w_{1}^{\prime}(t)}{D_{1}(t)}
\end{aligned}
$$




$$
\begin{aligned}
& \cdot \exp \left[j m t\left(\phi-\frac{\pi}{2}\right)\left(\frac{1}{2}+j \frac{\sqrt{3}}{2}\right)\right] d t \\
D_{1}(t)= & {\left[\zeta_{0} \exp \left(j \frac{\pi}{6}\right) w_{1}^{\prime}(t)+m \sin \theta_{0} w_{1}(t)\right] } \\
& \cdot\left[\eta_{0} \exp \left(j \frac{\pi}{6}\right) w_{1}^{\prime}(t)+m \sin \theta_{0} w_{1}(t)\right] \\
& +\zeta_{0} \eta_{0} m^{2} \cos ^{2} \theta_{0} w_{1}^{2}(t) \ldots \ldots \ldots \ldots \ldots \\
D_{2}(t)= & {\left[-j \zeta_{0} w_{2}^{\prime}(t)+m \sin \theta_{0} w_{2}(t)\right] } \\
& \cdot\left[-j \eta_{0} w_{2}^{\prime}(t)+m \sin \theta_{0} w_{2}(t)\right] \\
& +\zeta_{0} \eta_{0} m^{2} \cos ^{2} \theta_{0} w_{2}^{2}(t) \ldots \ldots \ldots \ldots .
\end{aligned}
$$

Kohei Hongo (Non-member) was born in Sendai, Japan, on July 2, 1939. He received B.S., M.S. and D.E. degrees in Electrical Communication Engineering from Tohoku University, Sendai, in 1962, 1964 and 1967, respectively. He joined the Faculty of Engineering, Shizuoka University, Hamamatsu, in April 1968, where he was a Professor until March 1991. In June 1992, he joined the Faculty of Science, and is currently a Professor of information science, Toho University, Funabashi, Japan. From 1967 to 1968 he was research associate of Electric Communication Engineering at Tohoku University. From 1991 to 1992, he was freelance consultant. His research interests include the application of the Kobayashi potential to diffraction problems associated with circular and rectangular conducting plates and more complex related configuration, and the refinement of physical optics for computing the field from the smooth and edged obstacles. He is a senior member of the IEEE.

Hirokazu Kobayashi (Non-member) was born in Hokkaido,

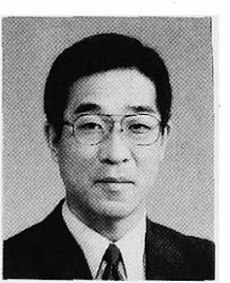
Japan , on April, 1955. He received B.E. and M.E. degrees from Shizuoka University, Hamamatsu, Japan, in 1978, 1980, respectively. He joined Fujitsu LTD., Kawasaki, Japan, in 1980. Since 1981 he has been engaging in research on microwave wide-band antennas, active phased array systems, and scattering cross-sections at the Fujitsu System Integration Laboratories. From 1991 he served as the manager of the microwave systems laboratory. His current research interests are high-frequency computing methods for large obstacles, especially based on physical optics. He is a member of the IEEE Antennas and Propagation Society. 